\title{
QUEEN'S
UNIVERSITY
BELFAST
}

\section{Speed of Saccadic Responses and Intelligence: An Exponential- Gaussian Analysis}

Wilson, P., Papageorgiou, K. A., \& Cooper, C. (2020). Speed of Saccadic Responses and Intelligence: An Exponential-Gaussian Analysis. Personality and Individual Differences, 158, [109860].

https://doi.org/10.1016/j.paid.2020.109860

\section{Published in:}

Personality and Individual Differences

\section{Document Version:}

Peer reviewed version

\section{Queen's University Belfast - Research Portal:}

Link to publication record in Queen's University Belfast Research Portal

\section{Publisher rights}

Copyright 2020 Elsevier

This manuscript is distributed under a Creative Commons Attribution-NonCommercial-NoDerivs License

(https://creativecommons.org/licenses/by-nc-nd/4.0/), which permits distribution and reproduction for non-commercial purposes, provided the author and source are cited.

\section{General rights}

Copyright for the publications made accessible via the Queen's University Belfast Research Portal is retained by the author(s) and / or other copyright owners and it is a condition of accessing these publications that users recognise and abide by the legal requirements associated with these rights.

Take down policy

The Research Portal is Queen's institutional repository that provides access to Queen's research output. Every effort has been made to ensure that content in the Research Portal does not infringe any person's rights, or applicable UK laws. If you discover content in the Research Portal that you believe breaches copyright or violates any law, please contact openaccess@qub.ac.uk. 
Personality and Individual Differences

Elsevier Editorial system(tm) for

Manuscript Draft

Manuscript Number: PAID-D-20-00133

Title: Speed of Saccadic Responses and Intelligence: An ExponentialGaussian Analysis.

Article Type: SI: PAID40 (Invited)

Section/Category: Research Paper (<5000 words)

Keywords: intelligence; cognition; eye movements; nerve conduction velocity

Corresponding Author: Dr. Colin Cooper, PhD

Corresponding Author's Institution: The Queen's University of Belfast

First Author: Paul Wilson, PhD

Order of Authors: Paul Wilson, PhD; Kostas A Papageorgiou, PhD; Colin Cooper, PhD

Abstract: Individual differences in the speed of making rapid eye movements (saccades) may have potential for exploring the link between neural conduction and cognitive abilities. Participants ( $N=56)$ performed tasks devised to measure the speed at which humans started to move an eye towards a stimulus which appeared in peripheral vision (saccade) or in the opposite direction (anti-saccade). Cognitive abilities were measured using the Wide Range Intelligence Test, and ex-Gaussian parameters from the eye-movement tasks were correlated with these abilities. The findings showed that scores on the pro- and anti-saccade tests correlated substantially and that the anti-saccade condition led to consistently longer and more variable and more skewed responses. None of the parameters correlated significantly with the cognitive abilities assessed. The findings do not support the theory that nerve conduction velocity explains the correlation between reaction times and cognitive abilities. However, the findings do provide evidence of the existence of individual differences in saccadic eye-movements that can be captured by ex-Gaussian analysis of reaction time and also show that saccadic movements do not follow Hick's Law when task difficulty is manipulated. 
Speed of Saccadic Responses and Intelligence: An Exponential-Gaussian Analysis.

Paul Wilson

Kostas A. Papageorgiou

Colin Cooper

School of Psychology

Queen's University

Belfast

BT7 1NN 


\section{Abstract}

Individual differences in the speed of making rapid eye movements (saccades) may have potential for exploring the link between neural conduction and cognitive abilities. Participants $(N=56)$ performed tasks devised to measure the speed at which humans started to move an eye towards a stimulus which appeared in peripheral vision (saccade) or in the opposite direction (anti-saccade). Cognitive abilities were measured using the Wide Range Intelligence Test, and ex-Gaussian parameters from the eyemovement tasks were correlated with these abilities. The findings showed that scores on the pro- and anti-saccade tests correlated substantially and that the anti-saccade condition led to consistently longer and more variable and more skewed responses. None of the parameters correlated significantly with the cognitive abilities assessed. The findings do not support the theory that nerve conduction velocity explains the correlation between reaction times and cognitive abilities. However, the findings do provide evidence of the existence of individual differences in saccadic eye-movements that can be captured by ex-Gaussian analysis of reaction time and also show that saccadic movements do not follow Hick's Law when task difficulty is manipulated. 


\section{Introduction}

There is a substantial literature linking cognitive abilities to reaction times, and it has been suggested that this may be because biological intelligence is linked to either the speed of neural conduction or the amount of "noise" introduced at the synapses (Eysenck, 1967; Jensen, 1993, 2006). Later studies of neural activity in the brain show that processing speed (as measured by inspection time and reaction time) completely explains the correlation between cerebral white matter tract integrity (Penke et al., 2012), showing that reaction time does indeed have a neural basis. However it is unclear whether reaction-time tasks measure anything as simple as speed of neural conduction; they could instead reflect individual differences in developing fast and efficient neural networks to process visual stimuli, which are known to be linked to general ability (Kim et al., 2016). Reaction time tasks may not be the pure measures of nerve conduction velocity as assumed by Eysenck and Jensen. It is thus useful to develop even simpler tasks and study whether latencies measured from these measures correlate appreciably with measures of $g$, as the neural speed theories would suggest.

The relationship between reaction time and $g$ is strongest when cognitive components are introduced; 8-choice rather than simple reaction times, or various elementary cognitive tasks (Sheppard \& Vernon, 2008). Although this can lead to experiments where the tasks used to measure reaction time resemble those which are found in tests which are commonly used to measure general ability (e.g., Luo \& Petrill, 1999). This clearly makes for a tautology; the more 
complex the task, the more likely it is that the same cognitive strategies/networks that are used in the ability tests will be used to solve the cognitive tasks. This is another reason to focus on low-level measures of reaction-time, rather as McRorie \& Cooper (2004) did when studying the link between reflex latencies and intelligence.

There are several other problems with the experimental designs which are most often used to measure reaction time. Most studies relating individual differences in reaction time to intelligence (e.g., Barrett, Eysenck, \& Lucking, 1986) correlate means and/or within-subject variability (standard deviations) with scores on ability tests, in an attempt to find whether speed of response or consistency of responding is linked to $g$. Unfortunately, these mean and standard deviations are substantially correlated as reaction times do not follow a normal distribution but instead show appreciable skew; a few long reaction times will inflate both an individual's mean and standard deviation. These studies thus cannot show whether speed or consistency explains the correlation between RT and $g$, an issue which can become important when trying to develop a theory of why this relationship arises.

The second difficulty is that tasks almost invariably involve pressing buttons with fingers, and hence measure a mixture of peripheral nerve activity and central nervous system (CNS) activity. Given that temperature (Rutkove, 2001), individual differences in myelinisation, and age (Verdu, Ceballos, Vilches, \& Navarro, 2000) are amongst the variables known to have a substantial influence on peripheral nerve conduction velocity it is possible that "noise" from the additional conduction involved from the peripheral nerves will mask any link between $g$ and 
nerve conduction velocity in the CNS, which might explain why reaction times typically show only modest correlations with measures of $g$.

Finally, not all studies attempt to differentiate movement time from reaction time, and those which attempt to do so (e.g., Jensen, 1982) generally use apparatus which requires rather gross movement of a heavy limb. Either of these approaches will probably introduce measurement error, given that movement times are uncorrelated with $g$ (Jensen \& Munroe, 1974).

The first of these problems can be solved by fitting an ex-Gaussian distribution to reaction time data (Luce, 1986) rather than using the mean and standard deviation. This approach estimates central tendency, variability and skew independently, and these three parameters can each be correlated with measures of $g$ (Osmon, Kazakov, Santos, \& Kassel, 2018). It seems that skew is a particularly important predictor of intelligence in traditional reaction-time tasks (Schmiedek, Oberauer, Wilhelm, Suss, \& Wittmann, 2007) as discussed below.

Studying eye movements may overcome the second and third problems, by providing a relatively pure measure of reaction time. The six extraocular muscles for each eye are powerful and are connected directly to the brainstem (Horn, Büttner-ennever, \& Büttner, 1996). The absence of peripheral nerves and slow motor responses means that saccades are much faster than simple reaction times, typically taking only $150 \mathrm{~ms}$ or so to initiate (Fischer \& Ramsperger, 1984 ) as opposed to some 500 ms for reaction times measured at the finger. Saccades are normally performed automatically whenever a stimulus appears in peripheral vision, and so 
responding to such a novel visual stimulus involves little conscious thought. The task is extremely low level, and may represent a relatively "pure" measure of nerve conduction velocity, uncontaminated by cognitive processes

Surprisingly perhaps there seems to have been no previous attempt to discover whether speed of making saccades is linked to $g$ or other cognitive abilities. Although Abdi Sargezeh et al., (2019) link general ability to looking patterns and variables such as peak saccade velocity, they did not measure the speed in which a saccade could be initiated when a stimulus appeared in the peripheral visual field.

It is possible to vary the cognitive load of tasks involving saccades. A participant might be warned to look at a shape which will be presented in either of two positions, or in one of four positions in the peripheral visual field, which is analogous to a two-choice or a four-choice reaction time task. A second way of increasing the cognitive load is by asking participants to suppress the saccade and look in the opposite direction to a stimulus presented at the periphery of the visual field - an "anti-saccade". Surprisingly, perhaps, inhibiting the saccadic response is difficult and frustrating but not impossible, and the neurological basis for inhibiting this response is well-understood (Munoz \& Everling, 2004).

The present study has three aims to address this gap in the literature by investigating individual differences in saccadic eye movements and their relationship with cognitive ability and add to the literature on mental chronometry and intelligence (Jensen, 2006). It seeks to determine 
whether any of the three ex-Gaussian parameters from tasks involving saccades show a stronger relationship to $g$ than is found in conventional reaction-time tasks involving finger movement. It determines whether altering the number of potential locations for a target on the periphery influences speed of response; in other words, whether Hick's Law describes saccadic responses. Finally, it determines whether the cognitively more demanding (and presumably slower) anti-saccadic responses show larger correlations with cognitive abilities than do the simpler tasks, just as cognitively complex cognitive tasks generally show larger correlations with abilities than do simple reaction times (Jensen, 2006).

\section{METHOD}

\section{Participants}

Following ethical approval, 60 participants were recruited by means of poster advertisements placed around the campus of a large UK university. However corruption of data files meant that usable data were available from only 56 people whose ages ranged from 17.5--45.0 (mean 21.8) years. It was subsequently discovered that two participants were not native English speakers, and their Verbal and FSIQ IQ data were discounted. Participants were screened for disorders of the nervous system (self-report) and for strabismus (squint). $25 \%$ were male. This design has a power of 0.75 of detecting a population correlation of 0.3 . 


\section{Apparatus and Materials}

As inhibitory control (the anti-saccade task) may have an effect on the development of both fluid and crystallised IQ according to investment theory it was important to use an intelligence assessment that provided measures of both subtypes of intelligence. The Wide Range Intelligence Test (WRIT; Glutting et al., 2000) provided standardised measures of verbal (crystallised), visual (fluid) and full scale IQs. Stinnett \& Widaman (2003:1014) describe the test's development as "gold standard" and correlations with the WAIS are above 0.85 (Glutting et al., 2000:97). Although the present sample was too small to allow an accurate estimate of alpha to be obtained, Glutting et al. (2000) reported coefficients alpha of the IQ scales for a similar age group to be 0.94 or greater.

An iView Hi-Speed Tracking Column (SensoMotoric Instruments $\mathrm{GmbH}$ ) was used to collect eye movement data 240 times per second from the left eye. It shone infrared light onto the face and used a camera to identify the position of the pupil. Saccades were defined as eye movements whose peak velocity of $40 \%$ s or above was reached within $20-80 \%$ of the saccade length, and which had a duration of $22 \mathrm{~ms}$ or longer.

Stimuli were presented using E-Prime 1.2 (Schneider, Eschman, \& Zuccolotto, 2002a, 2002b) on a Dell Optiplex GX620 PC running Microsoft Windows XP SP2 and a 16-inch Dell P793 colour monitor with a refresh rate of $75 \mathrm{~Hz}$. This system was linked to the eye tracking column to flag the onset time of each stimulus. Stimuli were presented in a black Ariel 18-point font against a white background. 


\section{Procedure}

The WRIT was administered individually, according to the test's standardised instructions; the procedure took approximately 45 minutes. Four eye-tracking tasks were completed in a second session at least 24 hours later to avoid fatigue. This session lasted approximately 50 minutes.

A pilot study showed that glare from spectacles and mascara both caused artefacts whereas contact lenses did not. Participants thus removed glasses and any mascara, and were asked to confirm that they could see the practice stimuli clearly. All reported they could perform the task comfortably and accurately without glasses. Participants were then seated and a chinrest was adjusted until the left eye was in the frame of the infra-red camera. Participants then looked at a circular target which was presented at 13 various locations on the screen. This calibrated the camera-recorded gazes to pre-determined screen positions. The procedure was repeated to check that the visual angle discrepancy was less than $0.5^{\circ}$, and it was repeated again if necessary.

Four tasks (four-choice pro-saccade; four-choice anti-saccade, two-choice pro-saccade; twochoice anti-saccade) were then administered in a counterbalanced fashion across participants to reduce any confounding effects on RT. Trial presentation within each task was randomised, with the same sequence of trials being presented to each participant to control for item-order effects (MacLeod, 1991). For each task there were ten practice trials, during which verbal 
feedback was provided by the experimenter, followed by 132 experimental trials for the fourchoice task, or 128 trials for the two-choice task. Participants were offered a break 25\%, 50\% and $75 \%$ of the way through each task, but usually chose to continue.

Each trial of a four-choice task presented a fixation cross in the centre of the screen which flashed three times (250ms on, $250 \mathrm{~ms}$ off) with a fourth cross having a random on-duration of either: $300,600,900$ or 1200 ms to avoid rhythmical response patterns (Jensen, 2006, pp. 4647). The fixation cross was extinguished at the same instant that the target stimulus (“@”) appeared at either the top left, top right, bottom left or bottom right of the screen for $500 \mathrm{~ms}$. The visual angle of the target from the fixation point was $23^{\circ}$, so ensuring that it was well into peripheral vision.

In the pro-saccade task, participants were instructed simply to look at the stimulus as quickly as possible when it appeared in peripheral vision, and that speed and accuracy were equally important. In the anti-saccade task they were asked not to look at the stimulus, but to look at the opposite corner as quickly as possible.

The two-choice task was similar, but stimuli were presented halfway up the left or right side of the screen. For the anti-saccade task participants were simply asked to shift their gaze horizontally to the other side of the screen. 


\section{Analysis}

The eye-tracking data were cleaned by rejecting any trials where gaze fixation was more than $2 \mathrm{~cm}$ from the fixation cross when the stimulus appeared, or where the eye movement in response to the stimulus was in the wrong direction. The latency of the initial saccade onset was taken to be the RT to each stimulus. Accuracy was calculated as the number of correct trials divided by the total number of valid trials. Individual RT distributions for each condition were screened for outliers, with reaction times which were more than four standard deviations higher than the individual's mean being rejected as recommended by Schmiedek, Oberauer, Wilhelm, Süß, and Wittmann (2007), by an iterative process. Reaction times below 100ms were regarded as pre-emptive and were also rejected. (Whelan, 2008). Model parameters were calculated using the program QMPE version 2.18 (Cousineau, Brown, \& Heathcote, 2004; Heathcote, Brown, \& Mewhort, 2002).

\section{Results}

A reliability analysis on the reaction time data showed that errors of measurement were small; coefficients alpha were greater than 0.99 for all four tasks. Likewise the correlations between the first half and the last half of each session were all greater than 0.99 , indicating that fatigue was unlikely to have affected the later trials. Table 1 shows the mean reaction times for the various conditions, together with IQ scores from the WRIT from the 2-choice tasks. 


\begin{tabular}{|l|l|l|l|l|}
\hline & N & Mean & sd & Min/Max \\
\hline Crystallised ability & 54 & 108.11 & 10.82 & $77 / 130$ \\
\hline Fluid ability & 56 & 110.43 & 11.50 & $82 / 133$ \\
\hline FSIQ & 54 & 110.35 & 11.10 & $86 / 134$ \\
\hline
\end{tabular}

Table 1.

Descriptive statistics from the Wide Range Intelligence Test 


\begin{tabular}{|l|l|l|l|l|l|l|}
\hline Task & N & Mean (sd) & Mu & Sigma & Tau & Mean \\
& & RT & & & accuracy \\
\hline 2-choice pro- & 56 & $0.22(0.10)$ & 0.188 & 0.020 & 0.031 & 0.98 \\
& & & $(0.097)$ & $(0.011)$ & $(0.014)$ & \\
\hline 2-choice anti- & 56 & $0.30(0.11)$ & 0.258 & 0.026 & 0.046 & 0.88 \\
saccade & & & $(0.103)$ & $(0.014)$ & $(0.027)$ & \\
\hline 4-choice pro & 54 & $0.23(0.10)$ & 0.197 & 0.020 & 0.036 & 0.95 \\
saccade & & & $(0.097)$ & $(0.012)$ & $(0.015)$ & \\
\hline 4-choice anti- & 54 & $0.34(0.13)$ & 0.292 & 0.034 & 0.054 & 0.79 \\
saccade & & & $(0.107)$ & $(0.029)$ & $(0.045)$ & \\
\hline
\end{tabular}

Table 2.

Descriptive statistics from the four saccade reaction time tasks, showing means and standard deviations together with the three ex-Gaussian parameters and accuracy. Mu = ex-Gaussian central tendancy component; Sigma = ex-Gaussain dispersion component.; Tau = ex-Gaussian skew component.

Parameter differences between pro and anti-saccade tasks were all in the expected direction with participants giving faster, more consistent RTs and less skewed reaction times in the prosaccade task. Wilcoxon tests showed that the differences between these conditions were highly significant. For the 2-choice condition the pro/anti-saccade differences for Mu, Sigma and Tau 
gave $W(56)$ values of 36,404 and 217 , all significant with Bonferroni $P<0.001$. Similar findings were found for the four-choice tasks: $W(54)=17,211$ and 385, all significant with Bonferroni $\mathrm{P}<0.002$.

\begin{tabular}{|l|l|l|}
\hline & 2-choice task & 4-choice task \\
\hline $\mathrm{Mu}$ & $0.698^{* *}$ & $0.704^{* *}$ \\
\hline Sigma & $0.442^{* *}$ & $0.416^{* *}$ \\
\hline Tau & $0.558^{* *}$ & $0.279^{*}$ \\
\hline
\end{tabular}

Table 3.

Spearman correlations between pro- and anti-saccade conditions for two-choice and fourchoice tasks. ${ }^{*} \mathrm{P}<0.05 * * \mathrm{P}<0.001$, one tailed.

The correlations in Table 3 shows the extent to which the three parameters of the ex-Gaussian distribution were consistent between the pro-and anti-saccade tasks. They are important because they show whether individuals who are slow to initiate saccades are also slow to inhibit them. 


\begin{tabular}{|c|c|c|c|c|}
\hline & & $\begin{array}{l}\text { Crystallised } \\
\text { ability }\end{array}$ & Fluid ability & FSIQ \\
\hline \multirow[t]{4}{*}{$\mathrm{Mu}$} & 2-choice pro-saccade & 0.112 & 0.045 & 0.110 \\
\hline & 2-choice anti-saccade & 0.031 & 0.002 & 0.063 \\
\hline & 4-choice pro saccade & 0.110 & 0.160 & 0.178 \\
\hline & 4-choice anti-saccade & -0.099 & 0.063 & 0.002 \\
\hline \multirow[t]{4}{*}{ Sigma } & 2-choice pro-saccade & 0.094 & -0.026 & 0.052 \\
\hline & 2-choice anti-saccade & -0.003 & 0.052 & 0.037 \\
\hline & 4-choice pro saccade & -0.086 & 0.072 & -0.016 \\
\hline & 4-choice anti-saccade & -0.288 & -0.011 & -0.195 \\
\hline \multirow[t]{4}{*}{ Tau } & 2-choice pro-saccade & 0.112 & 0.048 & 0.082 \\
\hline & 2-choice anti-saccade & 0.144 & -0.085 & 0.011 \\
\hline & 4-choice pro saccade & 0.157 & 0.127 & 0.149 \\
\hline & 4-choice anti-saccade & 0.064 & -0.153 & -0.053 \\
\hline
\end{tabular}

Table 4.

Pearson correlations between ex-Gaussian parameters and cognitive abilities 
None of the correlations between saccade initiation times and cognitive abilities were statistically significant by the Bonferroni criterion ( $\alpha=0.0042$ ).

\section{Discussion}

Table 3 Spearman correlations revealed a substantial link between people's performance on the pro-saccade and anti-saccade tasks. This indicates that there is consistency across conditions; the two tasks measure some aspect of individual differences. The Wilcoxon tests also show that the manipulation designed to increase cognitive workload through response inhibition (pro-vs anti-saccade condition) was effective; response times, response variability and skew were appreciably larger for the anti-saccade condition. These analyses are important, as they show that this novel methodology is tapping some aspect of individual differences rather than random noise, and that response times were longer for the more cognitively demanding condition, as expected. The methodology appears to be satisfactory.

It is therefore surprising that neither the speed (mu) or the variability (sigma) of people's saccadic reaction times was related to any of the ability measures. Tau reflected the degree of skew in each person's reaction time distribution. When choice reaction times are measured by pressing buttons, Hick's Law shows that reaction times become longer as the number of potential targets increases (Sheppard \& Vernon, 2008). Table 2 shows no hint of any difference in mu between the two-choice and 4-choice pro-saccades, or between the two- and four-choice 
anti saccade conditions. The time taken to initiate saccades does not appear to follow Hick's law.

Although requiring an anti-saccade rather than a saccade did slow response times as expected, the anti-saccadic tasks also showed no significant or appreciable correlation with any of the ability measures. This is in contrast with conventional measures of reaction time, where more cognitively demanding tasks generally show larger correlations with $g$ and other abilities than do simpler tasks, for the reasons discussed by Jensen (2006).

Analyses were also performed to determine whether the difference in ex-Gaussian parameters between the anti-saccade and pro-saccade conditions correlated with the ability measures; in other words, whether speed of the anti-saccade related to abilities after controlling for "baseline" saccadic latency. Two significant correlations were found, using Bonferroni corrections; the four-choice sigma (variability) correlated -0.236 with crystallised ability, and the 4-choice tau (skew) correlated -0.233 with fluid ability.

Given that the worst performance rule suggests that the slowest RTs of a distribution have larger correlations with IQ compared to the faster RTs, stronger correlations were expected between the tau parameter and IQ because this parameter represents the right-hand tail of the distribution (i.e. the slowest response times). Previous literature had also linked the tau parameter to attentional lapses (Heathcote et al., 1991; Kane \& Engle, 2003). It was expected that the tau parameter in the anti-saccade tasks would be strongly associated with an individual's information processing ability as measured by visual IQ: whereby higher ability should be associated with smaller tau values (i.e. fewer attentional lapses). Whilst the 
correlations between visual IQ and tau in the anti-saccade tasks were in the expected negative direction; the magnitude of the relationships were weak and failed to reach statistical significance at $\mathrm{P}<.05$. In general, the ex-Gaussian analysis of RT performance in the eye tracking tasks provided little support for any relationship between any form of cognitive ability and either level or change in ex-Gaussian parameters across pro and anti-saccade conditions. They provide little support for the hypothesis that speed of neural conduction is the reason why reaction times correlate with cognitive abilities.

\section{References}

Abdi Sargezeh, B., Ayatollahi, A., \& Daliri, M. R. (2019). Investigation of eye movement pattern parameters of individuals with different fluid intelligence. Experimental Brain Research, 237(1), 15-28. doi:10.1007/s00221-018-5392-2

Barrett, P. T., Eysenck, H. J., \& Lucking, S. (1986). Reaction time and intelligence - a replicated study. Intelligence, 10(1), 9-40.

Eysenck, H. J. (1967). Intelligence assessment: a theoretical and experimental approach. British Journal of Educational Psychology, 37, 81-98.

Fischer, B., \& Ramsperger, E. (1984). HUMAN EXPRESS SACCADES - EXTREMELY SHORT REACTION-TIMES OF GOAL DIRECTED EYE-MOVEMENTS. Experimental Brain Research, 57(1), 191-195.

Jensen, A. R. (1982). Reaction time and psychometric g. In H. J. Eysenck (Ed.), A Model for Intelligence. Berlin: Springer-Verlag.

Jensen, A. R. (1993). Spearman's g - links between psychometrics and biology. In F. M. Crinella \& J. Yu (Eds.), Brain Mechanisms: Papers in Memory of Robert Thompson (Vol. 702, pp. 103-129). New York: New York Acad Sciences. 
Jensen, A. R. (2006). Clocking the mind : mental chronometry and individual differences. Amsterdam ; London: Elsevier.

Jensen, A. R., \& Munroe, E. (1974). Reaction time, movement time and intelligence. Intelligence, 3, 121126.

Kim, D. J., Davis, E. P., Sandman, C. A., Sporns, O., O'Donnell, B. F., Buss, C., \& Hetrick, W. P. (2016). Children's intellectual ability is associated with structural network integrity. Neuroimage, 124, 550-556. doi:10.1016/j.neuroimage.2015.09.012

Luo, D. S., \& Petrill, S. A. (1999). Elementary cognitive tasks and their roles in g estimates. Intelligence, 27(2), 157-174. doi:10.1016/s0160-2896(99)00020-3

McRorie, M., \& Cooper, C. (2004). Synaptic transmission correlates of general mental ability. Intelligence, 32(3), 263-275.

Munoz, D. P., \& Everling, S. (2004). Look away: The anti-saccade task and the voluntary control of eye movement. Nature Reviews Neuroscience, 5(3), 218-228. doi:10.1038/nrn1345

Osmon, D. C., Kazakov, D., Santos, O., \& Kassel, M. T. (2018). Non-Gaussian Distributional Analyses of Reaction Times (RT): Improvements that Increase Efficacy of RT Tasks for Describing Cognitive Processes. Neuropsychology Review, 28(3), 359-376. doi:10.1007/s11065-018-9382-8

Penke, L., Maniega, S. M., Bastin, M. E., Hernandez, M. C. V., Murray, C., Royle, N. A., ... Deary, I. J. (2012). Brain white matter tract integrity as a neural foundation for general intelligence. Molecular Psychiatry, 17(10), 1026-1030. doi:10.1038/mp.2012.66

Rutkove, S. B. (2001). Effects of temperature on neuromuscular electrophysiology. Muscle \& Nerve, 24(7), 867-882. doi:10.1002/mus.1084

Schmiedek, F., Oberauer, K., Wilhelm, O., Suss, H. M., \& Wittmann, W. W. (2007). Individual differences in components their relations to working of reaction time distributions and memory and 
intelligence. Journal of Experimental Psychology-General, 136(3), 414-429. doi:10.1037/00963445.136.3.414

Sheppard, L. D., \& Vernon, P. A. (2008). Intelligence and speed of information-processing: A review of 50 years of research. Personality and Individual Differences, 44(3), 535-551. doi:10.1016/j.paid.2007.09.015

Verdu, E., Ceballos, D., Vilches, J. J., \& Navarro, X. (2000). Influence of aging on peripheral nerve function and regeneration. Journal of the Peripheral Nervous System, 5(4), 191-208. doi:10.1046/j.15298027.2000.00026.x 
Acknowledgements
\[ \begin{array}{l}\text { This research was funded by the Department for Employment } \\ \text { (Northern Ireland) through a PhD scholarship to the first author. }\end{array} \]

Acknowledgements
This research was funded by the Department for Employment and Learning
(Northern Ireland) through a PhD scholarship to the first author.

Acknowledgements
This research was funded by the Department for Employment a
(Northern Ireland) through a PhD scholarship to the first author.

(Northern lreland) through a PhD scholarship to the first author.

(1)
and Learning

(1)

(2)

(1) 